\title{
DOMINANT CLASSES OF PROJECTIVE VARIETIES
}

\author{
FEDERICO BUONERBA AND FEDOR BOGOMOLOV
}

\begin{abstract}
We give evidence for a uniformization-type conjecture, that any algebraic variety can be altered into a variety endowed with a tower of smooth fibrations of relative dimension one.
\end{abstract}

The problem of constructing a resolution of singularities of projective varieties is one of the most fundamental obstructions to our understanding of their analytic, arithmetic and geometric properties. A tremendous amount of work has shed light on the this problem, yet in its full generality it is still wide open over fields of positive characteristic. A cornerstone result, albeit conjecturally not optimal, is de Jong's dJ96 3.1, stating that any variety can be altered into a smooth projective one. Allowing alterations, other than birational modifications, comes along with a profusion of new natural questions, in the spirit of: which further properties can we require, for a class of smooth projective varieties, to be dominant? Recall from [BH00] that a class $\mathscr{C}(k, n)$ of $n$-dimensional projective varieties over a field $k$ is dominant if for every projective $n$-dimensional variety $X$, there exists $Y \in \mathscr{C}(k, n)$ and a surjective $k$-morphism $Y \rightarrow X$. In this terminology, de Jong's result says that smooth projective varieties form a dominant class over any field and in any dimension. Constructing minimal classes of dominant varieties is a problem that attracted attention, and a satisfactory answer is still unknown even in the case of curves over fields that are finitely generated over their prime subfield. Some results, questions and speculations in this direction can be found in [BT02, BT02], BT05. In this paper we give evidence for the following conjecture:

Conjecture 1 ([BH00]). For any field $k$ and positive integer $n$, the class of $n$-dimensional smooth projective varieties $X$, endowed with a tower of smooth fibrations

$$
X \rightarrow X_{1} \rightarrow \ldots \rightarrow X_{n}
$$

with $\operatorname{dim} X_{i}=n-i$, is dominant.

Section \will be devoted to the proof of: 
Theorem 1. The following classes $\mathscr{C}(k, n)$ are dominant:

(i) For $n=3$, smooth threefolds with a smooth connected morphism onto a smooth curve.

(ii) For any $n$ and $k$ a finite field, projective varieties admitting a connected morphism onto a smooth curve, with only one singular fiber whose singular locus consists of one ordinary double point.

Let us give a quick indication of the proof. The idea is to construct fibrations using Lefschetz pencils. In fact, the existence of Lefschetz pencils on smooth projective varieties, [SGA7.2 XVII, combined with de Jong's alteration result, dJ96] 3.1, immediately gives:

Fact. For any field $k$ and integer $n \geq 2$, the class of projective varieties admitting a connected morphism onto $\mathbf{P}_{k}^{1}$, with isolated singular fibers whose singular locus consists of one ordinary double point, is dominant.

Statement (i) of the Theorem is then an immediate consequence of the BrieskornTyurina's simultaneous resolution of surface ordinary double points, which in fact provides a simultaneous resolution of the fibers of the fibration induced by the Lefschetz pencil. Statement (ii) is more delicate. We are given $X$ a smooth and projective over a finite field, with $X^{\vee}$ the dual variety of singular hyperplane sections. We construct a curve $C$, in the space of hyperplanes of $X$, that intersects $X^{\vee}$ in a single point, which is a smooth point for both $X^{\vee}$ and $C$. In order to perform this construction, the crucial assumption, that $k$ is a finite field, manifests itself in that the Picard group - of degree zero divisor classes is always finite for projective curves. The total space of the induced family of hyperplane sections has a natural fibration onto $C$ with the required conditions on the fibers, and by construction it has a surjective morphism onto $X$.

In section 【I we focus our attention on surfaces. In this case the dual variety $X^{\vee}$ stratifies according to geometric genus of the generic member, and therefore one might try to understand the geometry and the modularity of such strata. The general idea is outlined in Proposition 10, The geometric structure of the stratification provides satisfactory answers for surfaces of negative Kodaira dimension, on which complete families of curves with constant geometric genus are easily constructed in Proposition 11, Such families induce, upon normalization of the total space, an equigeneric fibration with smooth general member. Unfortunately the situation becomes complicated in non-negative Kodaira dimensions, where the method doesn't provide any obvious answer on general K3's and hypersurfaces in $\mathbf{P}^{3}$ 
of degree at least 4 . The difficulty here is that the strata might be nested into each other as divisors, and it seems hard to insert a complete curve between two consecutive ones. Therefore we turn our attention to the modularity of the stratification. Less vaguely, each stratum $S^{k}$, the closure of the set of curves with geometric genus $g^{k}$, admits a rational map $S^{k} \rightarrow \overline{\mathscr{M}}_{g^{k}}$, and one might try to lift complete curves from $\mathscr{M}_{g^{k}}$ to $S^{k}$. Question 14 summarizes the difficulty with this approach, due essentially to the non-genericity of the image of this rational map, whose behaviour near the Deligne-Mumford boundary is, a priori, arbitrary.

In section III we continue our discussion by pointing out that the category of surfaces that can be dominated by complete families of smooth curves, which conjecturally is everything, is in fact rather flexible. By this we mean that it is somewhat natural and easy to create new smooth fibrations out of old ones, using ideas inspired by Kodaira's construction, [Ko67], of non-isotrivial smooth fibrations. First we show in Proposition [15, that any product of two curves can be dominated by a non-isotrivial smooth fibration. Finally we prove, in Proposition 17, that any two smooth fibrations can be dominated simultaneously by a third one.

In section IV, we conclude our discussion by analyzing several aspects of surfaces of general type that carry an everywhere smooth foliation. It turns out, Proposition 19, that such a surface must have positive topological index, which leads one to think about Kodaira fibrations. Indeed Brunella, in [Br97], has set the the foundations of the uniformization theory of such foliated surfaces, by establishing that the universal cover has the structure of a disk bundle over a disk. This, together with Corlette-Simpson's classification, CS08, of Zariski dense Kahler representations in $P S L_{2}(\mathbf{R})$ leads us to Theorem 21, a smoothly foliated surface of general type is either a Kodaira fibration, or a foliated subvariety of a polydisk quotient. It is extremely reasonable that, in the second case, our initial surface is itself a bidisk quotient.

We remark that the existence of ball and bidisk quotients can be used to prove, as in Proposition 22, that surfaces with an étale cover which is a Stein submanifold in a 3-dimensional ball form a dominant class.

Switching our attention to smooth foliations on surfaces over fields of characteristic $p>0$, the situation becomes as different from the complex case, as pleasant. Indeed we have: 
Theorem 2. Let $k$ be a field with $p:=\operatorname{char} k>2$, and let $X / k$ be an algebraic surface. Then there exists a birational modification of $X$, followed by an inseparable cyclic cover, such that the resulting surface $Y$ carries a smooth $p$-closed foliation.

It is worth remarking that the construction of $Y$ is extremely generic, in that we start with a general Lefschetz pencil in $X$, pick a general curve going through the nodes in the pencil, and finally take an inseparable cyclic cover branched along such curve. The foliation defining the Lefschetz pencil is shown to pull back, upon saturation, to a smooth and $p$-closed foliation, via a trivial local computation.

Due to the lack of Brunella's theorem in positive characteristic, the uniformization-type consequences that can be deduced from this statement, if any, are completely mysterious.

Acknowledgements We are deeply grateful to Michael McQuillan for his interest in this work, and for fundamental criticism of an attempt to prove the main conjecture over finite fields. We would also like to thank Misha Gromov, for several insights and pleasant conversations. The second author acknowledges that the article was prepared within the framework of a subsidy granted to the HSE by the Government of the Russian Federation for the implementation of the Global Competitiveness Program. The second author was partially supported by EPSRC programme grant EP/M024830, Simons Fellowship and Simons travel grant.

\section{Proof of theorem 1}

Let $X$ be a smooth projective variety of dimension $n$ over a field $k$, and let $\mathbf{P}_{X}$ denote the projective space of hyperplane sections of $X$, with universal family

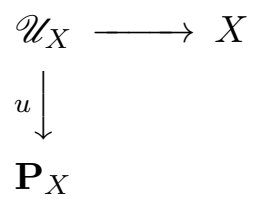

Let us prove (i). Assume $n=3$, and consider a Lefschetz pencil $f: \mathbf{P}_{k}^{1} \rightarrow \mathbf{P}_{X}$. There is a Zariski-closed subset $S \subset \mathbf{P}_{k}^{1}$ such that $\left(f^{*} \mathscr{U}_{X}\right)_{s}$ has a single ordinary double point if and only if $s \in S$. By the Brieskorn-Tyurina's simultaneous resolution of ordinary double points of surfaces, A74, there is a ramified cover $C \rightarrow \mathbf{P}_{k}^{1}$, and a birational morphism $Z \rightarrow C \times{ }_{\mathbf{P}_{k}^{1}} f^{*} \mathscr{U}_{X}$ such that the composite $Z \rightarrow C$ is a smooth morphism. 
What follows is the proof of (ii). Assume $k$ is a finite field, and denote by $X^{\vee} \subset \mathbf{P}_{X}$ the dual variety of singular hyperplanes. It is well known, SGA7.2 XVII, that upon replacing the projective embedding of $X$ with a multiple, $X^{\vee}$ is an irreducible divisor inside $\mathbf{P}_{X}$, whose smooth locus corresponds to hyperplane sections with a unique singular point, which is an ordinary double point.

Claim 3. In order to conclude the proof of the theorem, it is enough to find an irreducible curve $C \subset \mathbf{P}_{X}$ such that the intersection $C \cap X^{\vee}$ is supported in a single point, which is smooth for both $C$ and $X^{\vee}$.

Proof. Let $f: C \rightarrow \mathbf{P}_{X}$ be such curve, and let $u_{C}: f^{*} \mathscr{U}_{X} \rightarrow C$. $u_{C}$ has a unique singular fiber, whose singular locus is a single ordinary double point, lying over a smooth point of $C$. Therefore the induced fibration $f^{*} \mathscr{U}_{X} \times_{C} C^{\text {norm }} \rightarrow C^{\text {norm }}$ is the required one.

The rest of the proof will be devoted to the construction of such curve $C$. Let $S \stackrel{\sim}{\rightarrow} \mathbf{P}_{k}^{2}$ be a general linear plane inside $\mathbf{P}_{X}$, intersecting the divisor $X^{\vee}$ along an irreducible, reduced curve $D$. Denote by $d=\operatorname{deg}_{S}(D)$. Recall the well known

Fact 4. Since $k$ is finite, the group $\mathrm{Pic}_{D / k}^{0}$ of degree zero divisor classes on $D$ is finite.

Let $N>d$ be an integer that kills $\operatorname{Pic}_{D / k}^{0}$. Denote by $H$ an hyperplane section of $S$ and by $x$ a smooth point of $D$. By Fact 4 there exists an isomorphism of sheaves

$$
\mathscr{O}_{D}(N H) \stackrel{\sim}{\rightarrow} \mathscr{O}_{D}(M x)
$$

where of course $M=N d$, inducing a commutative diagram

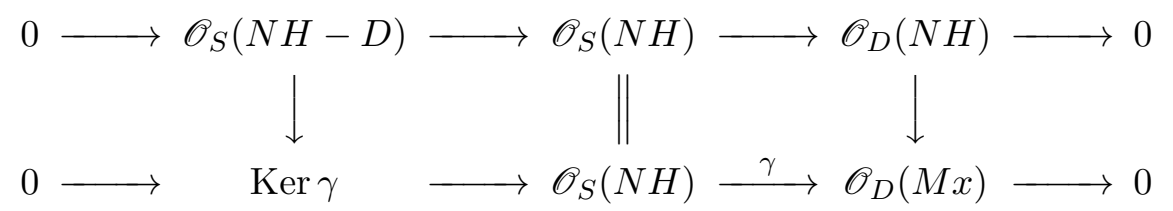

whose vertical arrows are all isomorphisms. By our choice of $N$ we deduce that $\operatorname{Ker} \gamma$ has non-trivial global sections, and the points of $\mathbf{P}\left(H^{0}(S, \operatorname{Ker} \gamma)\right)$ correspond to curves of degree $N$ inside $S$, whose intersection with $D$ is supported on $x$. All is left to do is to check that the generic member of the linear system $\mathbf{P}\left(H^{0}(S, \operatorname{Ker} \gamma)\right)$ is smooth at $x$. This is achieved by way of:

Lemma 5. Let $G, L \in k[X, Y, Z]$ be homogeneous polynomials of degrees $d$ and $N>d$ respectively. Assume that they define irreducible curves intersecting only at $x=[0: 0: 1]$ 
and that the curve defined by $G$ is smooth at $x$. If the curve defined by $L$ is singular at $x$, then $F=Z^{N-d} G+L$ satisfies

(i) the curves defined by $F$ and $G$ meet only in $x$, and

(ii) the curve defined by $F$ is smooth at $x$.

Proof. $\{x\}=\operatorname{Supp}((G=0) \cap(L=0))=\operatorname{Supp}((G=0) \cap(F=0))$, which is (i). In order to prove (ii), observe that $x$ is the origin of the affine space $Z=1$, so then denoting by $u=X / Z, v=Y / Z$ and $g(u, v)=Z^{-d} G(X, Y, Z), l(u, v)=Z^{-N} L(X, Y, Z)$ we see that $(F=0)$ is defined, around $x$, by the vanishing of $f=g+l$. Since $\nabla f(0,0)=\nabla g(0,0) \neq 0$ the proof is complete.

Consequently the generic member of $\mathbf{P}\left(H^{0}(S, \operatorname{Ker} \gamma)\right)$ is smooth at $x$, and can be taken to be the curve $C$ we are looking for.

\section{AN APPROACH TO THE CONJECTURE FOR SURFACES}

Let us describe some simple examples of surfaces for which Conjecture 1 holds:

Example 6. Minimal models of surfaces of negative Kodaira dimension: apart from $\mathbf{P}^{2}$, these are $\mathbf{P}^{1}$-bundles over smooth curves.

Example 7. Some surfaces of Kodaira dimension 0:

- Abelian varieties: Let $A$ be a d-dimensional abelian variety, and $f: C \rightarrow A$ any non-constant algebraic curve. The sum morphism $C^{d} \rightarrow A$ given by $\left(c_{1}, \ldots, c_{d}\right) \rightarrow$ $f\left(c_{1}\right)+\ldots+f\left(c_{d}\right)$ is surjective.

- Kummer K3 surfaces: Let $C$ be a genus 2 curve with hyperelliptic involution $\iota$. Consider the sequence of morphisms

$$
C \times C \rightarrow \operatorname{Sym}^{2} C \rightarrow \operatorname{Jac}^{2}(C),\left(c_{1}, c_{2}\right) \rightarrow c_{1}+c_{2} \rightarrow\left[c_{1}+c_{2}\right]
$$

The graph $\Gamma_{\iota}$ of the hyperelliptic involution is projected onto a rational curve by the first morphism, and it is contracted by the second. Pulling back the above diagram under a degree 16 cover $m_{2}: \operatorname{Jac}^{2}(C) \rightarrow \operatorname{Jac}^{2}(C)$ - obtained by identifying $\operatorname{Jac}^{2}(C) \stackrel{\sim}{\rightarrow} \operatorname{Pic}(C)$ via $K_{C}$ - we obtain morphisms

$$
m_{2}^{*}(C \times C) \rightarrow m_{2}^{*} \operatorname{Sym}^{2} C \rightarrow m_{2}^{*} \operatorname{Sym}^{2} C /(-1)=K 3(C)
$$

Since $m_{2}$ is étale, $m_{2}^{*}(C \times C)$ is still a product of curves. 
The previous example generalizes as follows:

Proposition 8. Let $k$ be a finite field, and $S_{0} \subset \mathbf{P}^{2}(k)$ any finite set. Then there exist curves $C_{1}, C_{2}$ and a morphism $C_{1} \times C_{2} \rightarrow \mathrm{Bl}_{\mathbf{P}^{2}}\left(S_{0}\right)$.

Proof. Observe that, for any such $S_{0}$, there exists a finite set $S \subset \mathbf{P}^{1}(k)$ and a morphism $\mathrm{Bl}_{\mathbf{P}^{1} \times \mathbf{P}^{1}}(S \times S) \rightarrow \mathrm{Bl}_{\mathbf{P}^{2}}\left(S_{0}\right)$. Let $E_{1}$ be an elliptic curve, and $C$ a curve of genus 2 with a non-constant morphism $C \rightarrow E_{1}$. We can assume wlog that the Jacobian of $C$ is isomorphic to $E_{1} \times E_{2}$, for some elliptic curve $E_{2}$. Consider non-constant morphisms $g_{i}: E_{i} \rightarrow \mathbf{P}^{1}$ and the resulting

$$
\left(g_{1}, g_{2}\right): \mathrm{Bl}_{E_{1} \times E_{2}}\left(g_{1}^{*} S \times g_{2}^{*} S\right) \rightarrow \mathrm{Bl}_{\mathbf{P}^{1} \times \mathbf{P}^{1}}(S \times S)
$$

By our assumption on $k$, any finite set of points in $E_{1} \times E_{2}$ is torsion, hence there exists an integer $N$ and a morphism

$$
m_{N}^{*} \mathrm{Bl}_{E_{1} \times E_{2}}(0 \times 0) \rightarrow \mathrm{Bl}_{E_{1} \times E_{2}}\left(g_{1}^{*} S \times g_{2}^{*} S\right)
$$

where $m_{N}$ is the multiplication by $N$ map on $E_{1} \times E_{2}$. Finally, composing

$$
C \times C \rightarrow \operatorname{Sym}^{2} C \stackrel{\sim}{\rightarrow} \mathrm{Bl}_{E_{1} \times E_{2}}(0 \times 0)
$$

with the morphisms constructed above, yields

$$
m_{N}^{*}(C \times C) \rightarrow m_{N}^{*} \mathrm{Bl}_{E_{1} \times E_{2}}(0 \times 0) \rightarrow \mathrm{Bl}_{E_{1} \times E_{2}}\left(g_{1}^{*} S \times g_{2}^{*} S\right) \rightarrow \mathrm{Bl}_{\mathbf{P}^{1} \times \mathbf{P}^{1}}(S \times S)
$$

In order to conclude, we employ [SGA1] X.1.7 to find curves $C_{1}, C_{2}$ and an isomorphism $C_{1} \times C_{2} \stackrel{\sim}{\rightarrow} m_{N}^{*}(C \times C)$.

Since every Hirzebruch surface is dominated by a blow-up, in a finite set of points, of $\mathbf{P}^{1} \times \mathbf{P}^{1}$, we obtain:

Corollary 9. Let $X$ be a Hirzebruch surface over a finite field, and $S \subset X(k)$ any finite set of points, then there exist curves $C_{1}, C_{2}$ and a morphism $C_{1} \times C_{2} \rightarrow \mathrm{Bl}_{X}(S)$.

Turning to a more general discussion, let $k$ be a field, $X / k$ a smooth projective surface, and $H$ an ample divisor such that $H^{1}\left(X, \mathscr{O}_{X}(n H)\right)=H^{2}\left(X, \mathscr{O}_{X}(n H)\right)=0$ for all $n \geq 1$. We have the linear system $\mathbf{P}_{X, n}=\mathbf{P}\left(H^{0}\left(X, \mathscr{O}_{X}(n H)\right)\right)$ of dimension

$$
d_{n}=\operatorname{dim}\left(\mathbf{P}_{X, n}\right)=\operatorname{dim} H^{0}\left(X, \mathscr{O}_{X}(n H)\right)-1=n H \cdot\left(n H-K_{X}\right) / 2+\chi\left(\mathscr{O}_{X}\right)-1
$$


and its generic member is smooth of genus $g_{n}=1+n H \cdot\left(n H+K_{X}\right) / 2$. Observe that $\mathbf{P}_{X, n}$ admits a natural stratification

$$
\emptyset=: S_{n}^{N(n)+1} \subsetneq S_{n}^{N(n)} \subsetneq \ldots \subsetneq S_{n}^{0}:=\mathbf{P}_{X, n}
$$

by, not necessarily irreducible, closed subvarieties, such that the generic member of each irreducible component of $S_{n}^{k}, 0 \leq k \leq N(n)$, corresponds to a curve with geometric genus at most $g_{n}^{k}$, and of course $0 \leq g_{n}^{N(n)}<\ldots<g_{n}^{0}=g_{n}$. We will call it the Severi stratification. For $g_{n}^{k} \geq 2$ there is rational map

$$
p: S_{n}^{k} \rightarrow \overline{\mathscr{M}}_{g_{n}^{k}}
$$

into the Deligne-Mumford compactification of the moduli stack of curves. The reason to introduce the Severi stratification is:

\section{Proposition 10.}

(i) Assume that, for some $0 \leq k \leq N(n)$ such that $g_{k} \geq 2$, there exists a smooth proper curve $C$ and a non-constant morphism $f: C \rightarrow S_{n}^{k}$ such that the rational map pof $: C \rightarrow \overline{\mathscr{M}}_{g_{n}^{k}}$ extends, after a finite cover $C^{\prime} / C$, to a morphism $C^{\prime} \rightarrow \mathscr{M}_{g_{k}}$. Then, upon replacing $C$ by a finite cover, there exists a smooth surface $Y$, a smooth fibration $Y \rightarrow C$, and a surjective morphism $Y \rightarrow X$.

(ii) For some $0 \leq k \leq N(n)$, there exists a smooth proper curve $C$ and a non-constant morphism $f: C \rightarrow S_{n}^{k} \backslash S_{n}^{k+1}$. Then, upon replacing $C$ by a finite cover, there exists a smooth surface $Y$, a fibration $Y \rightarrow C$ whose fibers have constant geometric genus, and a surjective morphism $Y \rightarrow X$.

Proof.

(i) As in the previous section, $\mathbf{P}_{X, n}$ is equipped with a universal space $u: \mathscr{U}_{X, n} \rightarrow$ $\mathbf{P}_{X, n}$, and we can consider the fibration $u_{C}: f^{*} \mathscr{U}_{X, n} \rightarrow C$. The normalization $u_{C}^{\text {norm }}:\left(f^{*} \mathscr{U}_{X, n}\right)^{\text {norm }} \rightarrow C$ induces the moduli morphism $p \circ f: C \rightarrow \overline{\mathscr{M}}_{g_{k}}$ and therefore after replacing $C$ by $C^{\prime}$, the resulting fibration is smooth.

(ii) Since the curves in our family $u_{C}$ are equigeneric - meaning that the geometric genus is constant along the fibers - we have that $u_{C}^{\text {norm }}$ is again an equigeneric family, with smooth general member. 
The natural problem becomes to investigate to what extent Proposition 10 can be applied. The next proposition shows how the geometry of the moduli $\mathscr{M}_{3}$ can be used to construct complete families of smooth curves mapping to the plane, using 10,(i):

Fact. There exists a complete family $p: Y \rightarrow C$ of smooth genus 3 curves, whose fibrewise canonical map $\left|p_{*} \omega_{Y / C}\right|: Y \rightarrow C \times \mathbf{P}_{k}^{2}$ realizes the generic curve as a smooth quartic, and the special ones as double conics with smooth support.

Proof. The Torelli map $\mathscr{M}_{3} \rightarrow \mathscr{A}_{3}$ is bijective on closed points, so there is a canonical Baily-Borel compactification $\mathscr{M}_{3}^{B B}$, which is projective and with boundary in codimension 2. Therefore, a generic complete intersection curve $C \subset \mathscr{M}_{3}^{B B}$ is contained in $\mathscr{M}_{3}$ and intersects the hyperelliptic locus $\mathscr{H}_{3} \subset \mathscr{M}_{3}$ transversely. Moreover the canonical map $\left|\omega_{C}\right|: C \rightarrow \mathbf{P}_{k}^{2}$ realizes points of $\mathscr{H}_{3}$ as plane double conics, and points of $\mathscr{M}_{3} \backslash \mathscr{H}_{3}$ as smooth plane quartics.

We refer to [HM98], pp.133 for a discussion around the modular behavior of families plane quartics degenerating to double concics.

Something can be said on surfaces with negative Kodaira dimension, indeed 10. (ii) quickly proves:

Proposition 11. Let $X$ be a smooth surface with negative Kodaira dimension. Then for $n$ sufficiently big, there exists a smooth proper curve $C$ and a non-constant morphism $f: C \rightarrow S_{n}^{k} \backslash S_{n}^{k+1}$. Therefore, $X$ can be dominated by an equigeneric family of curves.

Proof. Consider the following inductive construction: set $S_{0}:=S_{n}^{0}$, and assuming defined $S_{k}$, let $S_{k+1} \subset S_{k}$ be an irreducible component of $S_{n}^{k+1}$ of maximal dimension. We claim that, for some $k, \operatorname{dim} S_{k}-\operatorname{dim} S_{k+1} \geq 2$ : by assumption the canonical bundle of $X$ is not pseudoefective, therefore $K_{X} \cdot H<0$, so then $d_{n}-g_{n}=-n H \cdot K_{X}-2+\chi\left(\mathscr{O}_{X}\right)$ is positive for $n$ sufficiently big. Hence it is impossible to have $\operatorname{dim} S_{k}=\operatorname{dim} S_{0}-k=d_{n}-k$ for every $k$.

Remark 12. Unfortunately, this is still not enough to prove the conjecture in negative Kodaira dimension: obviously, there exist equigeneric families of curves with smooth generic member, yet carrying singular, necessarily not irreducible, members.

The situation becomes more interesting in non-negative Kodaira dimension, where the genus $g_{n}$ tends to be bigger than the dimension $d_{n}$, and the stratification might consist of strata of consecutive codimension one. In fact this happens on general hypersurfaces: 
Theorem 13. [CC98] Let $X$ be a general hypersurface of $\mathbf{P}^{3}$ of degree $d \geq 4$. For $n \geq d$ and any $0 \leq k \leq d_{n}$, the variety $S_{n}^{k}$ contains an irreducible component of dimension $d_{n}-k$ whose generic point parametrizes curves with $k$ nodes.

In this situation, the best we can hope in order to construct a smooth family is a positve answer to:

Question 14. Can we find $n, k$ such that the boundary divisor $\Delta_{g_{n}^{k}} \subset \overline{\mathscr{M}}_{g_{n}^{k}}$, restricted to the closure of $p\left(S_{n}^{k}\right)$, is not ample? Even better, admitting a contraction?

A positive answer to the above would provide us with a curve to which apply Proposition 10 (i), and hence prove the conjecture in dimension 2. The problem is that the image of $p$ in the moduli $\mathscr{M}_{g_{n}^{k}}$ is going to be of high codimension and extremely non-generic. For example, consider the natural rational map

$$
p: \overline{\mathscr{M}}_{g_{n} \rightarrow \mathscr{M}_{g_{n}}^{B B}}
$$

Then, unless $X$ is dominated by an isotrivial surface, $\overline{p\left(S_{n}^{k}\right)} \cap \Delta_{g_{n}^{k}}$ is not contracted by $p$, albeit $p$ does restrict to a contraction along $\Delta_{g_{n}^{k}}$.

\section{FLEXIBILITY OF KODAIRA FIBRATIONS}

In this section we emphasize that the class of Kodaira fibrations, i.e. those surfaces admitting a non-isotrivial smooth morphism onto a smooth curve, is remarkably flexible, and there plenty of smooth fibrations that can be constructed out of given ones. First, let us review Kodaira's original construction, [Ko67]: given any curve $C_{0}$ of genus at least 2, let $C \rightarrow C_{0}$ be any non-trivial, finite étale cover with Galois group $\Gamma$. Consider, for any $m \||\Gamma|$, the natural quotient $\pi_{1}(C) \rightarrow H_{1}(C, \mathbf{Z} / m \mathbf{Z})$, and the corresponding étale cover $f: C^{\prime} \rightarrow C$ with Galois group $H_{1}(C, \mathbf{Z} / m \mathbf{Z})$. The crucial observation is that, by the Kunneth formula, the class of the graph $\Gamma_{f}$ inside $H^{2}\left(C^{\prime} \times C, \mathbf{Z} / m \mathbf{Z}\right)$ depends uniquely on the morphism $f^{*}: H^{q}(C, \mathbf{Z} / m \mathbf{Z}) \rightarrow H^{q}\left(C^{\prime}, \mathbf{Z} / m \mathbf{Z}\right)$. By construction, this morphism is trivial when $q=1,2$, while it is an isomorphism when $q=0$. In particular, the cohomology class of $\Gamma_{\gamma \circ f}, \gamma \in \Gamma$ is independent of $\gamma$. Since $m|| \Gamma \mid$, we deduce that $D:=\cup_{\gamma} \Gamma_{\gamma \circ f}$ is $m$ divisible in $H^{2}\left(C^{\prime} \times C, \mathbf{Z}\right)$. Let $X=X(C, m) \rightarrow C^{\prime} \times C$ be the cyclic covering of order $m$, branched along $D$. Since the $\Gamma_{\gamma \circ f}$ are pairwise disjoint and each of them is an étale multisection of the second projection $p_{2}: C^{\prime} \times C \rightarrow C$, we deduce that the composition 
$X \rightarrow C$ is a non-isotrivial smooth fibration.

We now employ Kodaira's construction as follows:

Proposition 15. Given two curves $C_{1}, C_{2}$, there exists a curve $C$, a smooth non-isotrivial fibration in smooth curves $Y \rightarrow C$ and a finite morphism $Y \rightarrow C_{1} \times C_{2}$.

Proof. Let $C_{0}$ be a curve of genus at least 2 with two surjective morphisms $f_{i}: C \rightarrow C_{i}$. Running the above construction, we obtain a Kodaira fibration $X$, with a natural sequence of finite morphism $X \rightarrow C^{\prime} \times C \rightarrow C \times C \rightarrow C_{1} \times C_{2}$.

The class of surfaces of general type that are finite quotients of products of curves is vast. [BCF15], and references therein, point to a detailed study of the class of so-called product-quotient surfaces, which are, by the previous proposition, also dominated by nonisotrivial smooth fibrations.

Similarly to what has been done in Proposition [15, let $C$ of genus $g \geq 2$, and consider the divisor $\Delta_{m} \subset \operatorname{Sym}^{m} C$ inside the $m$-fold symmetric product of $C$, of points $\left(c_{1}, \ldots, c_{m}\right)$ with $c_{i}=c_{j}$ for some $i \neq j$.

Proposition 16. Let $f: D \rightarrow \operatorname{Sym}^{m} C \backslash \Delta_{m}$ be a non-constant morphism from a complete curve $D$. Then there exists a smooth non-isotrivial fibration $Y \rightarrow D$ and a finite morphism $Y \rightarrow C \times D$.

Proof. The morphism $f$ defines a divisor $D_{f} \subset C \times D$, whose fiber over $d \in D$ is the $m$-tuple of points $f(d) \subset C$, and by construction the projection $p: D_{f} \rightarrow D$ is étale. As before, upon replacing $D$ by a non-trivial Galois cover, one constructs Kodaira fibrations as cyclic covers of $D_{f} \times D$, branched along the Galois orbit of the graph of $p$.

Observe that this construction provides many examples when $m=2$, since $\Delta \subset \mathrm{Sym}^{2} C$ can be contracted via $\operatorname{Sym}^{2} C \rightarrow \operatorname{Jac}^{0}(C) /(-1),\left(c_{1}, c_{2}\right) \rightarrow\left[c_{1}-c_{2}\right]$. The case $m \geq 3$ is more subtle, since the diagonal $\Delta_{m} \subset \operatorname{Sym}^{m} C$ lies on the boundary of the effective cone of $\mathrm{Sym}^{m} C$, and even its numerical properties seem rather mysterious.

In a deeper vein, the next proposition shows that pairs of Kodaira fibrations admit common refinements, meaning that they can dominated simultaneously by a third Kodaira fibration.

Proposition 17. Let $q_{1}: X_{1} \rightarrow C_{1}$ and $q_{2}: X_{2} \rightarrow C_{2}$ be smooth fibrations in curves. Then there exists a smooth curve $C_{0}$ with surjective morphisms $t_{i}: C_{0} \rightarrow C_{i}$, a fibration in curves $X \rightarrow C_{0}$ and finite $C_{0}$-morphisms $X \rightarrow t_{i}^{*} X_{i}, i=1,2$. 
Proof. We first fix some notation: for a given curve $D$, denote by $\mathscr{M}_{g}(D)$ the substack of $\mathscr{M}_{g}$ parametrizing smooth genus $g$ curves $C$ that admit a surjective morphism $C \rightarrow D$. Similarly, for a given pair of curves $D_{1}, D_{2}$, denote by $\mathscr{M}_{g}\left(D_{1}, D_{2}\right)$ the substack of $\mathscr{M}_{g}$ parametrizing smooth genus $g$ curves $C$ that admit surjective morphisms $C \rightarrow D_{1}$ and $C \rightarrow D_{2}$. The above admits a relative analogue, in that if $\mathscr{D}_{1} \rightarrow B$ and $\mathscr{D}_{2} \rightarrow B$ are two families of curves, we have an induced fibration $\mathscr{M}_{g}\left(\mathscr{D}_{1}, \mathscr{D}_{2} / B\right) \rightarrow B$, whose fiber over $b \in B$ is $\mathscr{M}_{g}\left(\mathscr{D}_{1, b}, \mathscr{D}_{2, b}\right)$. A trick by Kodaira, HM98] 2.33, shows that for every $n$, there exists $g$ such that $\mathscr{M}_{g}(D)$ contains complete subvarieties of dimension $n$, and therefore the same holds for $\mathscr{M}_{g}\left(D_{1}, D_{2}\right)$.

We are now ready to offer a proof of the proposition: let $C$ be a curve with surjective morphisms $t_{i}: C \rightarrow C_{i}$ and replace $q_{i}$ by $p_{i}: Y_{i}:=t_{i}^{*} X_{i} \rightarrow C$. Consider the fibration $\pi: \mathscr{M}_{g}\left(Y_{1}, Y_{2} / C\right) \rightarrow C$. By the above remarks, for $g$ big enough there exists a surface

$Z \subset \mathscr{M}_{g}\left(Y_{1}, Y_{2} / C\right)$ such that $\pi_{\mid Z}: Z \rightarrow C$ is surjective and the fiber $Z \cap \pi^{-1}(c)$ is a complete curve for generic $c \in C$. Such morphism $\pi_{\mid Z}$ clearly admits a multisection $C_{0}$ for example by taking an ample divisor in $\bar{Z}$, the closure of $Z$ in $\overline{\mathscr{M}}_{g}\left(Y_{1}, Y_{2} / C\right)$, missing the isolated boundary points - and such multisection $C_{0}$ induces, by definition, a smooth fibration in curves $X \rightarrow C_{0}$ dominating $Y_{1} / C$ and $Y_{2} / C$.

\section{SMooth FOLiations on SURFACES OF GENERAL TYPE}

Motivated by the study of surfaces that carry smooth fibrations, we dedicate this final section to surfaces of general type carrying smooth foliations. In particular we look for restrictions the existence of a smooth foliation imposes on the ambient surface, and then try to understand what they might possibly look like. Recall that a smooth foliation $\mathscr{F}$ on an algebraic surface $X$ defines an exact sequence of vector bundles

$$
0 \rightarrow T \mathscr{F} \rightarrow T X \rightarrow N \mathscr{F} \rightarrow 0
$$

The next fact recollects some well known numerical properties of $K \mathscr{F}:=T \mathscr{F}^{\vee}$.

\section{Fact 18.}

(i) $c_{2}(X)=K \mathscr{F} \cdot\left(K_{X}-K \mathscr{F}\right)$.

(ii) $\left(K_{X}-K \mathscr{F}\right)^{2}=0$.

(iii) $c_{2}(X)=K_{X}^{2}-K \mathscr{F} \cdot K_{X}$.

(iv) If $X$ is of general type then $K \mathscr{F}$ is pseudoeffective, and it is big unless $\mathscr{F}$ is either an isotrivial fibration, or a Hilbert modular foliation. 
Proof.

(i) This follows by taking Chern numbers in the defining exact sequence of $T \mathscr{F}$, plus $c_{2}(X)>0$.

(ii) This is the Baum-Bott index formula, $N \mathscr{F}^{2}=0$.

(iii) This is a formal consequence of (i) and (ii).

(iv) The pseudoeffectivity of $K \mathscr{F}$ follows from the Main Theorem of [BM16], since $X$ has general type. By the birational classification of foliations on surfaces, McQ08, if a foliation on a surface of general type is such that $K \mathscr{F}$ is not big, then the foliation is an isotrivial or Hilbert modular.

Therefore, granted a decent amount of understanding of isotrivial and Hilbert modular surfaces, we concentrate on the strong topological and algebraic restrictions, for a surface of general type to carry a smooth foliation.

Proposition 19. Let $X$ a smooth surface of general type, and $\mathscr{F}$ an everywhere smooth foliation on $X$ with $K \mathscr{F}$ big. Then we have

- $\operatorname{rank} \operatorname{Pic}(X) \geq 2$.

- $X$ has positive topological index, i.e. $c_{1}(X)^{2}>2 c_{2}(X)$.

Proof. Let $a:=\frac{K_{X} \cdot K \mathscr{F}}{K_{X}^{2}}$, and $R:=a K_{X}-K \mathscr{F}$. Since $R \cdot K_{X}=0$ we deduce, by Hodge index theorem, that $R^{2}<0$ unless $R$ is numerically trivial.

Claim 20. $R \neq 0$

Proof. We have

$$
0=R \cdot K_{X}=-K \mathscr{F} \cdot K_{X}+K_{X}^{2}+(a-1) K_{X}^{2}=c_{2}(X)+(a-1) K_{X}^{2}
$$

so then

$$
c_{2}(X)=(1-a) K_{X}^{2}
$$

If $R=0$ then $a K_{X}=K \mathscr{F}$, and $\left(K_{X}-K \mathscr{F}\right)^{2}=0$ implies $a=1$, from which $c_{2}(X)=0$, impossible since $X$ has general type.

From which we deduce that $\operatorname{rank} \operatorname{Pic}(X) \geq 2$.

Let $P \subseteq N S(X)$ be the plane spanned by $K_{X}$ and $R$. We know that

$$
\operatorname{Amp}(X)=\left\{D: D^{2}>0, D \cdot K_{X}>0\right\}
$$


so then Fact 18 (i) and (ii) imply that $K_{X}-K \mathscr{F}=(1-a) K_{X}+R$ lies on the boundary of $\operatorname{Amp}(X) \cap P$. It follows that $\operatorname{Amp}(X) \cap P$ is bounded by the rays

$$
(1-a) K_{X}+R,(1-a) K_{X}-R
$$

Since

$$
K \mathscr{F}=a K_{X}-R \in \operatorname{Amp}(X) \cap P
$$

by assumption, we have $a>1-a$, or $a>1 / 2$. The identity

$$
c_{2}(X)=(1-a) K_{X}^{2}
$$

derived in the proof of Claim [20, concludes the proof.

More interestingly, Brunella [Br97] has initiated the uniformization theory on smoothly foliated surfaces $(X, \mathscr{F})$ of general type: the universal cover $\tilde{X}$ of such a surface, admits a smooth holomorphic fibration $p_{\mathscr{F}}: \tilde{X} \rightarrow \Delta$ onto the unit disk, with disks as fibers, such that $\mathscr{F}$ becomes tangent to $p_{\mathscr{F}}$ on $\tilde{X}$. In particular, $X$ is a $K(\Gamma, 1)$, for $\Gamma:=\pi_{1}(X)$. With the aim of better understanding the geometry of $X$, observe that the fibration $p_{\mathscr{F}}$ is naturally $\Gamma$-equivariant, and hence there is a natural representation $\rho: \Gamma \rightarrow P S L_{2}(\mathbf{R})$. Corlette and Simpson, in CS08, have given a complete classification of what such a $\rho$ can be, and deduced a beautiful dichotomoy for its geometric origin. We can summarize all of this in:

Theorem 21 (Brunella, Corlette \& Simpson). Let $(X, \mathscr{F})$ be a smoothly foliated surface of general type. Then at least one of the following happens:

- $X$ admits a smooth fibration $p: X \rightarrow C$ onto a smooth curve $C$ and $\mathscr{F}$ is tangent to $p$;

- $\rho$ is rigid and integral, there exists a quasiprojective polydisk quotient, $Y$, and a natural morphism $X \rightarrow Y$ such that $\mathscr{F}$ is induced by one of tautological codimension one foliations on $Y$.

The only issue to be settled here, is the relation between the dimensions of $X$ and $Y$. It seems plausible, thinking about the wild behaviour of Hilbert modular foliations, that indeed the dimensions must be the same, and $X$ itself is a bidisk quotient.

Let us remark that bounded symmetric domains can be used in our problem of finding dominant classes as follows: 
Proposition 22. Let $X$ be a smooth algebraic surface. Then there exists a finite morphism $Y \rightarrow X$, such that an étale cover $Y^{\prime}$ of $Y$ is a Stein submanifold of a 3-dimensional ball.

Proof. Let $Z$ be a ball quotient, consider projections $X \rightarrow \mathbf{P}^{2}, Z \rightarrow \mathbf{P}^{2}$, and let $Y:=$ $X \times_{\mathbf{P}^{2}} Z$. For sufficiently generic projections, the branch loci in $\mathbf{P}^{2}$ are transverse, hence $Y$ is smooth. Let $p: \mathbf{B}^{2} \rightarrow Z$ be the universal cover, then $Y^{\prime}:=p^{*} Y$ is a complex manifold which is naturally a finite ramified cover of the ball $\mathbf{B}^{2}$, and as such embeds into a product $\mathbf{B}^{2} \times \Delta$.

We wish to conclude by turning our attention to the corresponding problem in characteristic $p>0$, of understanding smooth foliations on algebraic surfaces. Perhaps not too surprisingly, the situation is drastically different from the complex case, and it turns out that it is extremely simple to construct a covering of a given surface that carries a smooth foliation. Before proceeding to our main result, let us recall a key difference between foliations in characteristic 0 and $p$, that lies in the notion of integrability: the celebrated Frobenius integrability theorem, as well known for integrable distributions over $\mathbf{C}$, fails over fields of positive characteristic: integrable distributions need not have a formal first integral. Observe, indeed, that the notion of leaf is rather badly behaved, and the closest we can get to formal integrability is $p$-closedness - that is, the algebra generated by the vector fields defining our foliation is closed under $p$-powers. This implies that the kernel of such algebra of differential operators defines a factorization of the Frobenius morphism on the ambient variety. And a modicum of thought shows that this is the best integrability condition one can hope for. In practice, probably the quickest way of appreciating the role of $p$-closedness in remedying the failure of formal integrability is:

Fact 23. McQ08, II.1.6] Let A be a complete regular local ring over an algebraically closed field of characteristic $p$, and let $\partial$ be a non-singular derivation of $A$. Then there exists a regular system $x, y_{1}, \ldots, y_{n}$ of parameters such that

$$
\partial=\frac{\partial}{\partial x}+x^{p-1} \sum_{i=1}^{n} f_{i}\left(x^{p}, y\right) \frac{\partial}{\partial y_{i}}
$$

Moreover the ideal defining the vanishing of $\partial \wedge \partial^{p}$ is generated by $f_{1}, \ldots, f_{n}$. In particular $\partial$ is $p$-closed iff $\partial=\frac{\partial}{\partial x}$, i.e. a smooth foliation by curves is $p$-closed iff it is formally integrable. 
IV.1. Proof of Theorem 2, Let $p: Y_{0} \rightarrow \mathbf{P}_{k}^{1}$ be a general Lefschetz pencil in $X$, whose general member is smooth, and whose singular members have exactly one node. Observe that $Y_{0}$ is obtained by blowing up $X$ along the base points of the pencil. Let $S \subset \mathbf{P}_{k}^{1}$ parametrize the singular fibers, and for each $s \in S$ let $y_{s} \in Y_{s}$ be the node in the fiber. Let $H$ be a very ample divisor, and consider the sheaf $\mathscr{O}(p H) \otimes I_{\cup_{s} y_{s}}$ whose local sections are those of $\mathscr{O}(p H)$ vanishing along $\cup_{s} y_{s}$. Its generic global section defines a curve, $D$, which is smooth, transverse to the branches of $Y_{s}$ at $y_{s}$, and has simple tangencies with the fibers of $p$ outside their nodes. The following claim will conclude:

Claim 24. Let

$$
r_{D}: Y:=Y_{0}(\sqrt[p]{D}) \rightarrow Y_{0}
$$

denote the inseparable cyclic p-cover, branched along $D$. If $\mathscr{F}$ denotes the foliation defined by $p$, then the saturation of $r_{D}^{*} \mathscr{F}$ is smooth and $p$-closed.

Proof. First we deal with smoothness. We need to worry about what happens at:

(i) The nodes in the fibers of $p$, and

(ii) The simple tangencies between $D$ and the smooth locus of the fibers of $p$.

Let us consider (i). In the local ring of $Y_{0}$ completed in a node in a singular fiber, the branches of the singular fiber give us local coordinates $x, y$, while $D$ is defined by the vanishing of a third local function, $z$. $\mathscr{F}$ is defined by the vanishing of the form $d(x y)$, and our assumptions on $D$ imply that there exists a formal function $G$ such that $y=G(x, z)$ holds. The cyclic cover $r_{D}$ is defined by $r_{D}^{*} z=z^{p}$, hence

$$
r^{*} d(x y)=d\left(x \cdot G\left(x, z^{p}\right)\right)=\left(G+x \frac{\partial G}{\partial x}\right) d x
$$

and therefore, upon saturation, $r_{D}^{*} \mathscr{F}$ is smooth around the pre-image, under $r_{D}$, of nodes in the fibers of $p$.

Let us deal with (ii). In the local ring of $Y_{0}$ completed in a point of simple tangency, we can pick local coordinates $x, y$ such that our fibration is defined by the vanishing of the form $d y$. If the vanishing of $z$ defines $D$ in such coordinates, simple tangency means that there exists a formal function $g$ such that $z=y-g(x)$ and $\operatorname{ord}_{x} g(x)=2$.

In particular, $\frac{d g(x)}{d x}$ is not identically zero, since $p>2$.

The cyclic cover $r_{D}$ is defined by

$$
r_{D}^{*} z=z^{p}, r_{D}^{*} x=x, r_{D}^{*} y=z^{p}+g(x)
$$


and the pullback foliation is then

$$
r_{D}^{*} d y=d\left(z^{p}+g(x)\right)=\frac{d g(x)}{d x} d x
$$

which is again smooth upon saturation.

Observe, as an output of our local computations, that the saturation of $r_{D}^{*} \mathscr{F}$ is not only smooth, but also formally integrable. By Fact 23, it is $p$-closed.

\section{REFERENCES}

[A74] M. Artin, Algebraic construction of Brieskorn's resolutions, J. Alg., Vol. 29, Issue 2, May 1974, pp. 330-348, http://www.sciencedirect.com/science/article/pii/0021869374901021

[BCF15] I. Bauer, F. Catanese, D. Frapporti, The fundamental group and torsion group of Beauville surfaces, Beauville surfaces and groups, 114, Springer Proc. Math. Stat., 123, Springer, Cham, 2015. 14Fxx (14J25), http://arxiv.org/abs/1402.2109

[BH00] F. Bogomolov, D. Husemoller, Geometric properties of curves defined over number fields, MPI preprint , 2000-1, http://www.mpim-bonn.mpg.de/preprints

[BM16] F. Bogomolov, M. McQuillan, Rational curves on foliated varieties, Foliation Theory in Algebraic Geometry. Springer International Publishing, 2016. 21-51, http://link.springer.com/content/pdf/10.1007\%2F978-3-319-24460-0_2.pdf

[BT02] F. Bogomolov, Y. Tschinkel, Unramified correspondences, Algebraic number theory and algebraic geometry, Contemp. Math., vol. 300, Amer. Math. Soc., Providence, RI, 2002, pp. 17-25, http://arxiv.org/abs/math/0202223

[BT02'] F. Bogomolov, Y. Tschinkel, On curve correspondences, Communications in Arithmetic Fundamental Groups and Galois Theory, RIMS, pp.157-166, 2002, http://www.math.nyu.edu/ tschinke/papers/yuri/02genram/genram.pdf

[BT05] F. Bogomolov, Y. Tschinkel, Couniformization of curves over number fields, Geometric methods in algebra and number theory, pp. 43-57, Progress in Math. 235, Birkhuser, 2004 , http://www.math.nyu.edu/ tschinke/papers/yuri/04covers/cover4.pdf

[Br97] M. Brunella, Feuilletages holomorphes sur les surfaces complexes compactes, Annales scientifiques de l'École Normale Supérieure (1997) Volume: 30, Issue: 5, page 569-594 http://archive.numdam.org/ARCHIVE/ASENS/ASENS_1997_4_30_5/ASENS_1997_4_30_5_569_0/ASENS_1997_4_30_5

[CC98] L. Chiantini, C. Ciliberto, On the Severi varieties of surfaces in $\mathbf{P}^{3}$, http://arxiv.org/abs/math/9802009

[CS08] K. Corlette, C. Simpson, On the classification of rank-two representations of quasiprojective fundamental groups, Compos. Math. 144 (2008), 12711331, https://arxiv.org/pdf/math/0702287.pdf

[SGA7.2] P. Deligne, N. Katz, Séminaire de Géométrie Algébrique du Bois Marie - 1967-69 - Groupes de monodromie en géométrie algébrique - (SGA 7) - 
vol. 2. LNM 340. Berlin, New York: Springer-Verlag. pp. $\mathrm{x}+438, \quad 1973$, https://publications.ias.edu/sites/default/files/Number12.pdf

[dJ96] A.J. de Jong, Smoothness, semi-stability and alterations, Publications Mathématiques de l'IHÉS 83, 1996, pp. 51-93, http://www.math.uiuc.edu/K-theory/0081

[SGA1] A. Grothendieck, Séminaire de Géométrie Algébrique du Bois Marie - 1960-61 - Revêtements étales et groupe fondamental - (SGA 1). LNM 224. Springer-Verlag. pp. xviii+327, 1971, https://arxiv.org/abs/math/0206203

[HM98] J. Harris, I. Morrison, Moduli of curves, Graduate Texts in Mathematics, 187. Springer-Verlag, New York, 1998. xiv+366 pp, http://link.springer.com/content/pdf/10.1007\%2Fb98867.pdf

[Ko67] K. Kodaira, A certain type of irregular algebraic surfaces Journal d'Analyse Mathematique, vol. 19 (1967), pp. 207-215

[McQ08] M. McQuillan, Canonical models of foliations, Pure Appl. Math. Q. 4 (2008), no. 3, part 2, 8771012, http://www.mat.uniroma2.it/ mcquilla/files/canmod.pdf

FEDERICO BUONERBA

Courant Institute of Mathematical Sciences, New York University, 251 Mercer Street, New York, Ny 10012, USA

E-mail address: buonerba@cims.nyu.edu

Fedor Bogomolov

Courant Institute of Mathematical Sciences, New York University, 251 Mercer Street, New York, Ny 10012, USA National Research University Higher School of Economics, Russian Federation, AG Laboratory, hSE, 7 Vavilova str., Moscow, Russia, 117312

E-mail address: bogomolo@cims.nyu.edu 